$l$-galactose residues, although at the time of the publication by Jones and Peat' ${ }^{2}$ this was perhaps a natural assumption. The ethereal sulphate could indeed be situated on $\mathrm{C}_{3}$, since recent experiments in this laboratory have shown that methylglucofuranoside-3sulphates yield $3: 6$-anhydrides on alkaline hydrolysis. It may be added further that if the hydroxyl groups on $\mathrm{C}_{3}$ were thus esterified, the agar would possess no $\alpha$-glycol groupings, and would therefore not react with periodic acid, thus providing an alternative explanation for the results of Barry and Dillon². It should be noted, however, that this suggestion that the sulphate residue might be found on $\mathrm{C}_{3}$ is not put forward as a rival hypothesis to that of Jones and Peat ${ }^{1}$, but as a possible alternative, to emphasize the fact that additional work on agar specimens of known history is needed before further progress can be made in determining the structure of this polysaccharide.

King's Buildings,

University of Edinburgh.

\footnotetext{
1 Jones and Peat, J. Chem. Soc., 225 (1942).

2 Barry and Dillon, Chem. and Ind., 63, 167 (1944).

${ }^{3}$ Pereival and Thomson, J. Chem. Soc., 750 (1942)

4 Hands and Peat, Chem. and Ind., 57, 937 (1938).

- Forbes and Percival, J. Chem. Soc., 1844 (1939).

'Duff and Percival, J. Chem. Soc., 830 (1941).
}

\section{Non-conservative Fundamental Particles}

IN view of certain possibilities, for example the still open possibility of the non-existence of the neutrino, it may be interesting to set up an equation for a fundamental particle which, in the restricted relativity approximation, does not obey the energy conservation law.

Such a non-conservation property is easily arrived at by taking a Lagrangian which depends explicitly on the four co-ordinates. It is remarkable, however, that such a Lagrangian, or alternatively the corresponding equations, are almost automatically obtained, in an elegant way, by starting from the Dirac form and merely taking full account of the Lorentz invariance. The simplest form of equation is obtained as follows. Let us start from the Dirac equation

$$
\gamma^{\mu} \partial_{\mu} \psi+k \psi=\dot{0} \quad\left(\partial_{\mu}=\partial / \partial x^{\mu}\right) .
$$

It is well known that the Lorentz invariance is not altered if one adds a term of the form $A_{\mu \nu} \gamma^{\mu} \gamma^{\nu}, A_{\mu \nu}$ being an antisymmetric tensor of second rank. The simplest tensor of this type attached to the particle is obviously $x_{\mu} \partial_{\nu}-x_{\nu} \partial_{\mu}$. In order to make it invariant also under a change of origin, we take $\left(x_{\mu}-b_{\mu}\right) \partial_{\nu}-\left(x_{\nu}-b_{\nu}\right) \partial_{\mu}, b_{\mu}$ representing the coordinates of a point in space-time, the simplest case again being $b_{\mu}=$ constant. Putting therefore $m^{\mu \nu}=i / 2 \cdot\left(\gamma^{\mu} \gamma^{\nu}-\gamma^{\nu} \gamma^{\mu}\right)$, the new equation is, in the case of no field :

(1) $\gamma^{\mu} \partial_{\mu} \psi+i \lambda\left(x_{\sigma}-b_{\sigma}\right) m^{\sigma \mu} \partial_{\mu} \psi+k \psi=0$

$(\lambda=$ real constant).

It is obvious that dual terms in $\gamma^{\rho} \gamma^{\sigma} \gamma^{\tau}$ and $\gamma^{1} \gamma^{2} \gamma^{3} \gamma^{4}$ could also be added without disturbing the invariance; but as only the contribution of the $\gamma^{\mu} \gamma^{\nu}$ term brings something new, we shall leave them out at the present stage.

The particle defined by (1) has some remarkable properties close to those of Dirac's electron. It has a constant charge, and we may therefore call it a 'particle'. Its main feature is the spontaneous change of energy and momentum. The total amount of energy varies, even in the case of no field and even if the particle is 'at rest' and therefore has a vanishing kinetic energy. As the time passes, the particle loses or gains energy, even when resting, just like, for example, a living organism does. This change is connected with the existence of the new term in the equation and is proportional to the 'age' of the particle and to the constant $\lambda$. It can be computed from the expression of the symmetrical energymomentum tensor ${ }^{1} T^{\mu \nu}$, which leads to :

$$
\partial_{\nu} T^{\mu \nu}=2 \lambda\left(x^{\mu}-b^{\mu}\right)\left(\partial_{\rho} \psi^{*} i \gamma^{4} m^{\sigma \rho} \partial_{\sigma} \psi\right),
$$

giving thus a physical interpretation of the constant $\lambda$.

To define such a particle one must give, apart from its mass, a constant $\lambda$ and a point in spacetime $b_{\mu}$, intrinsically defined as the (chosen) point around which the particle is almost conservative, behaving, in fact, like a classical Dirac electron. To achieve the definition of a particle by giving not only a mass and $\lambda$, but also a point in space-time, is most unfamiliar but by no means physically absurd. One can picture, for example, a conservative particle bound to some system and escaping from it at a given moment. If, when escaping from the nucleus (or in general when being created), the particle acquires the non-conservation character, the moment and place where this happens are indeed an essential feature of its definition.

The symmetry of $T \mu \nu$ causes the total angular momentum at the fixed point $b_{\mu}$ to be constant.

$\lambda$ is not necessarily small, so that the supplementary term cannot be always considered as a small perturbation. In this case one gets interesting results by investigating what happens around a certain point $x^{k}=a^{k}$ at a definite instant $x^{4}=a^{4}$, that is by assuming that $a^{\mu}=$ const. The equation is then solved by a plane wave $\psi=A \exp i\left(p_{\mu} x^{\mu}\right)$, where $p_{\mu}$ is proportional to the energy and momentum.

The classical relation $(W / c)^{2}=\overrightarrow{p^{2}}+w^{2} c^{2}$ between energy and momentum is no longer valid; the new relation shows that, in the Newtonian approximation, the total energy is composed of the kinetic energy arising from the translation movement of the particle, plus a rotation energy, a fact which can be foreseen from the original form of the equation.

Unlike Dirac's case, the quantum mechanical treatment of this particle is essentially one which uses an indefinite metric in Hilbert space ${ }^{2}$, as the normalization integral is

$$
\int \psi^{*}\left[1+\lambda\left(x_{k}-b_{k}\right) \gamma^{k}\right] \psi d \tau \text {. }
$$

The Hamiltonian form $\partial \psi / \partial t+i H \psi=0$ is easily obtained and brings to light the discontinuity sphere $1-\lambda^{2} \Sigma\left(x_{x}-b_{x}\right)^{2}=0$, which plays an important part in the discussion and gives a geometrical interpretation of the newly introduced constant $\lambda$.

The striking fact about the above equation is that it follows quite naturally from a straightforward application of restricted relativity to the problem of the electron. This does not mean that it necessarily fits the physical reality, but seems to point towards the fact that its study might be worth while.

I am indebted to Prof. P. A. M. Dirac for invaluable discussion.

26 Cornwall Gardens,

A. Proca.

London, S.W.7.

Oct. 11.

${ }^{1}$ Cf. Portugalice Physica, 1, 159 (1943).

${ }^{2}$ Cf. Pauli, Rev. Mod. Phys., 15, 176 (1943). 